\title{
Mesiodistal Bone Loss of Fresh Socket Placed Implants and Delay Placed Implants at the Anterior of the Maxilla
}

\author{
Reza Tabrizi, Mohammadreza Azizi
}

\begin{abstract}
Introduction: Immediate implant placement in fresh extraction sockets provides the advantage of shorter treatment time along with minimizing the number of surgical interventions.
\end{abstract}

Materials and methods: Subjects were studied in two groups: in group 1, subjects received a fresh socket placed implant at the anterior of the maxilla and in group 2, a delay placed implant was inserted at the same site. The mesial and distal marginal bone level was measured using parallel technique radiography in time 0, 12, 24 and 36 months after loading.

Results: Seventy subjects were studied in two groups. Analysis of the data using a repeat measure test demonstrated the similar bone loss patterns between two groups. Time has a significant effect on bone loss in time 1, 2, 3. The amount of bone loss increased as time passed after loading time. Comparison of bone loss in both groups demonstrated that the amount of bone loss in the central site was more than the lateral site in three measurement times for the group 2 but in the group 1, a significant difference was not observed.

Conclusion: Comparison of marginal bone loss in fresh socket placed implants and delay implants did not show a significant difference. Also a continuous bone resorption was observed over the time in the both groups. It may be needed to investigate other success criteria in fresh socket placed implants for making an appropriate treatment plane.

Keywords: Implant, Fresh socket, Bone resorption, Maxilla, Immediate.

How to cite this article: Tabrizi R, Azizi M. Mesiodistal Bone Loss of Fresh Socket Placed Implants and Delay Placed Implants at the Anterior of the Maxilla. Int J Oral Implantol Clin Res 2013;4(2):63-67.

\section{Source of support: Nil}

Conflict of interest: None declared

\section{INTRODUCTION}

Implant placement in fresh extraction sockets has become increasingly routine, and surgical protocols have been modified, with a shift from the belief that total bone regeneration in the socket was thought to be required before implant placement to the common opinion that the best bone-preserving method is immediate implant placement. ${ }^{1}$ Some concerns related to immediate implant placement have been expressed and an early placement was rather suggested. This means that a time period of 4 to 6 weeks after tooth extraction is maintained to let the soft tissue heal. This facilitates soft tissue management at the time of the implant placement and leads to more predictable treatment outcomes, which is important in esthetically demanding sites. $^{2}$

In fact, immediate implant placement in fresh extraction sockets provides the advantage of shorter treatment time along with minimizing the number of surgical interventions. This reduces patient's morbidity and increases his comfort. Some authors also suggested biologic and esthetic advantages because the risk of alveolar bone resorption after tooth extraction could be reduced, and the gingival and crestal bone architecture will be better maintained. ${ }^{3,4}$ Additionally, a positive effect can be expected if immediately placed implants are immediately restored with provisional reconstructions. $^{5}$

A systemic review study demonstrated the high survival rate of implants in fresh socket extraction. ${ }^{6}$ However, a few studies reported on success rates rather than survival rates in the literature reviews. Short-term clinical results were described and results were comparable to those obtained with delayed implant placement. ${ }^{7}$

The amount of crestal bone resorption may not be predictable and there is a risk of inadequate implant position in the socket, particularly a buccal position will compromise esthetics of anterior maxillary implants. ${ }^{8}$

Nevertheless, because of the lack of long-term data, questions regarding whether peri-implant health, prosthesis stability, degree of bone loss, and esthetic outcome of immediate or early placed implants are comparable with implants placed in healed sites, remain unanswered. ${ }^{9}$

The aim of the present study was to compare the amount of mesiodistal bone loss of fresh socket placed implants and delay placed implants at the anterior of the maxilla.

\section{MATERIALS AND METHODS}

This is a prospective study to evaluate the amount of mesiodistal bone loss of fresh socket placed implants (group 1) and a delay placed implants (group 2) from Sep 1, 2008, to Oct 31, 2009. The research committee of the medical ethics group of Shiraz Medical Science University approved this study. Subjects eligible for group 1 had a fresh socked at central or lateral incisor of the maxilla due to avulsion or 
extraction and required restoration with an implant. Indications for extraction of failing teeth were endodontic failure, root fracture of teeth with posts and extensive caries lesions. Subjects were excluded from group 1 if they lost the tooth because of periodontal problem or had an infected tooth, periapical lesion and bone defect on buccal bone. Subjects were candidate for group 2 who lost a tooth at central or lateral incisor of the maxilla more than 3 months. The subjects who needed bone augmentation were removed from the group 2. None of the subjects in both the groups had systemic diseases that affected bone metabolism and severe parafunctional habits.

Each subject received an implant in the central or lateral site of the maxilla. All subjects' implants were loaded 3 months after insertion. One type of implant (Internal hex, RBT body, BioHorizons, USA) was used in both groups. The size of fixture was $3.8 \times 12 \mathrm{~mm}$. The follow-up time was 3 years after loading for both groups. None of the patients had a soft tissue or hard tissue manipulation during the follow-up period. Cemented crowns were used in all subjects.

The method of assessing the bone resorption and marginal bone level on the mesial and distal surfaces of the implants was a long-cone paralleling technique. The patients were radiographed with standard periapical film (no. 2, type E) with exposure parameters of $70 \mathrm{kV}, 8 \mathrm{~mA}$, and 0.250 seconds.

To confirm the reproducibility of the radiographs (after surgery and follow-up radiography), the radiographs were taken with individual bite blocks attached to the beamguiding device (XCP, Rinn, Elgin, IL). To individualize the bite blocks, bite registrations were fabricated using silicon impression material (Polyvinylsiloxane, Kerr, Germany) and placed on the individual bite blocks. A 5.5 $\mathrm{mm}$ spherical metal bearing was placed on the buccal surface of the neighboring tooth as a reference guide for measuring the magnification factor. The films were digitized using a digital scanner at an input resolution of 2,400 dots/inch and a 256 Gray (Gy) scale. The images of the teeth were enlarged 6 times and analyzed by a maxillofacial radiologist using Adobe Photoshop 5 (Adobe, San Jose, CA). The bone level was measured on the mesial and distal surfaces of each implant, and the fixture was measured on paired radiographs from the holder of the implant to the crest of the alveolar bone in the vertical dimension. Radiographs were taken immediately after loading (time 0), 12 months (time 1), 24 months (time 2) and 36 months (time 3) after loading. The bone level at the time of implant loading was defined as the baseline for the evaluation of marginal bone resorption. Three bone level changes (time 1-time 0 , time 2-time 1 and time 3-time 2) were used for evaluation of bone loss.

\section{Surgical Approach}

First local anesthesia (lidocaine 2\% with epinephrine $1 / 80,000,1.8-2.7 \mathrm{ml}$ ) was injected by infiltration route. After local anesthesia, the teeth or broken roots were carefully removed to avoid fracture of the socket walls and traumatic pressure. The socket walls were explored with a probe to recognize defects particularly of the buccal wall, which would be a contraindication for the immediate implant placement in the present study. The implant was placed along the palatal wall in the in anterior zone of the maxilla, because a more vertical direction of the prospective implant axis was aimed in comparison with the tooth axis. The implant length mostly exceeded the height of the root socket and the normal drilling protocol was applied, but the implant itself was inserted manually with a ratchet at a maximum of $30 \mathrm{Ncm}$. The implant shoulder was placed $2 \mathrm{~mm}$ below of the buccal bone crest, that was, approximately 3 to $5 \mathrm{~mm}$ underneath the soft tissue border of the socket. Autogenous bone graft was used when the crestal space between the implant and the incongruent socket walls was more than $2 \mathrm{~mm}$.

Because a fully closed suture was often not obtained, the socket above the implant was additionally covered with Gelatamp (Coltene/Whaledent, Langenau, Germany) to avoid a direct exposure of the implants to the oral cavity. In the group 2, after anesthesia a full-thickness crestal incision, slightly buccal but still within attached mucosa, was performed and extended mesially and distally to the adjacent teeth with leaving 1.0 to $1.5 \mathrm{~mm}$ of the interproximal papilla adjacent to each tooth. Two vertical incisions were made to enhance surgical access. Drilling was performed according to factory suggested protocol and an implant was inserted manually as the group 1 procedure, $1 \mathrm{~mm}$ under of the buccal bone crest.

\section{STATISTICAL ANALYSES}

Statistical analyses were performed using SPSS 19 for the PC (IBM, New York, NY). Chi-square test was used to compare sex and tooth site (central and lateral) between two groups. An independent t-test was applied to find a difference of age between two groups and to evaluate bone loss between two sites. Repeat measure test was used to evaluate bone loss in three measurement times (time 1: 12 months, time 2: 24 months, time 3: 36 months) after loading.

\section{RESULTS}

We studied 70 subjects in two groups. Group 1 consisted of 35 subjects who received an implant in a fresh extracted 


\begin{tabular}{|c|c|c|c|}
\hline \multicolumn{4}{|c|}{ Table 1: Comparison of variable factors between two groups } \\
\hline Variable factors & Group 1 & Group 2 & $p$-value \\
\hline Age & $36.43 \pm 13.84$ & $35.40 \pm 12.97$ & $>0.05^{\star}$ \\
\hline Sex & $\begin{array}{l}18 \text { (male) } \\
17 \text { (female) }\end{array}$ & $\begin{array}{l}16 \text { (male) } \\
19 \text { (female) }\end{array}$ & $>0.05^{\star *}$ \\
\hline Implant site & $\begin{array}{c}26 \text { (central) } \\
9 \text { (lateral) }\end{array}$ & $\begin{array}{l}23 \text { (central) } \\
12 \text { (lateral) }\end{array}$ & $>0.05^{\star \star}$ \\
\hline
\end{tabular}

*Independent t-test; ${ }^{*}$ Chi-square test

Table 2: Bone loss in the lateral and the central sites in the
group 1
\begin{tabular}{llll} 
Study time & \multicolumn{2}{c}{ Bone loss $(\mathrm{mm})$} & Independent $t$-test \\
\cline { 2 - 3 } & Central & Lateral & \\
\hline Time 1 & $0.59 \pm 0.23$ & $0.46 \pm 0.16$ & $\mathrm{p}>0.05$ \\
Time 2 & $0.72 \pm 0.25$ & $0.65 \pm 0.21$ & $\mathrm{p}>0.05$ \\
Time 3 & $0.83 \pm 0.32$ & $0.72 \pm 0.19$ & $\mathrm{p}>0.05$ \\
\hline
\end{tabular}

Table 3: Bone loss in the lateral and the central sites in the group 2

\begin{tabular}{llll}
\hline \multirow{2}{*}{ Study time } & \multicolumn{2}{c}{ Bone loss $(\mathrm{mm})$} & Independent $t$-test \\
\cline { 2 - 3 } & Central & Lateral & \\
\hline Time 1 & $0.70 \pm 0.18$ & $0.51 \pm 0.15$ & $\mathrm{p}<0.05$ \\
Time 2 & $0.84 \pm 0.17$ & $0.57 \pm 0.14$ & $\mathrm{p}<0.05$ \\
Time 3 & $0.98 \pm 0.18$ & $0.70 \pm 0.12$ & $\mathrm{p}<0.05$ \\
\hline
\end{tabular}

socket in the anterior of the maxilla. Thirty five subjects who underwent a delay implant procedure in the anterior of the maxilla were studied in the group 2. Eighteen males and 17 females with the mean age $36.43 \pm 13.84$ years involved in the group 1 and 16 males and 19 females with mean age of $35.40 \pm 12.97$ years were studied in the group 2 (Table 1). Comparison of the mean age of subjects did not show a significant difference between two groups ( $\mathrm{p}$ $>0.05$ ). Two groups did not have any significant difference of subjects' sex ( $>0.05)$. In group 1, 26 implants were placed in the central site and nine implants in the lateral site, in group 2, 23 implants were inserted into the central site and 12 implants into the lateral site. Evaluation of the data did not show a statistically difference of implant sites ( $p>0.05$ ). Analysis of the data using a repeat measure test demonstrated the similar bone loss patterns between two groups (Graph 1). Time has a significant effect on bone loss in time1, 2 and 3. The amount of bone loss increased as time passed after loading time. Comparison of bone loss in both groups demonstrated that the amount of bone loss in the central site was more than the lateral site in time 1, time 2 and time 3 for the group 2 but in the group 1, a significant difference was not observed (Tables 2 and 3).

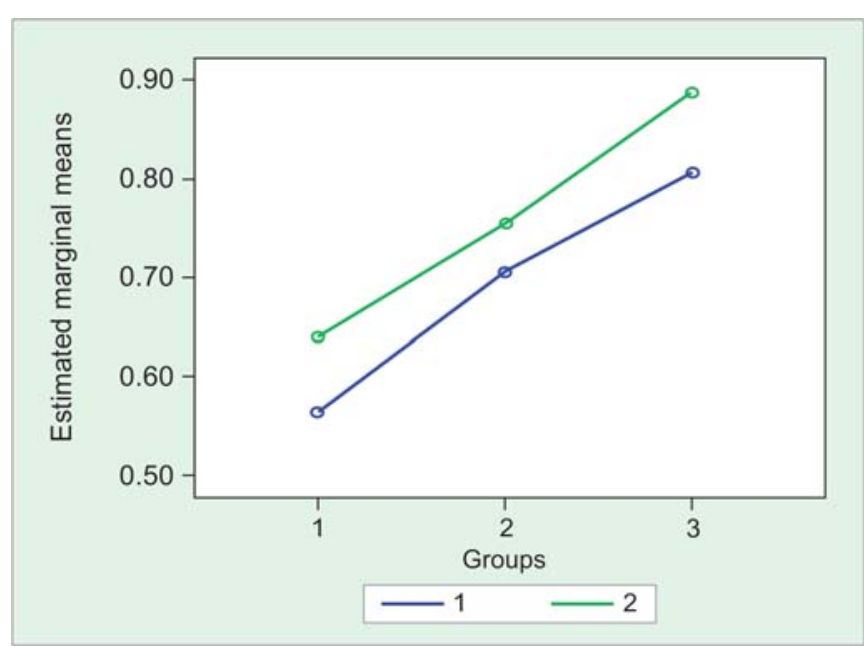

Graph 1: Comparison of bone loss $(\mathrm{mm})$ in three measurement times between two groups

\section{DISCUSSION}

After placing an implant in a fresh socket, it is expected that bone resorption happen as a part of bone healing. Also, remodeling of the alveolar crest after extraction follows a pattern, with resorption and reshaping of the alveolar crest. This marginal resorption is, of course, time dependent: the longer the healing time, the greater the resorption. ${ }^{10}$ Nowadays, to maintain bone height and achieve more rapid rehabilitation, immediate placement of implants in conjunction with extraction is commonly practiced in many clinics. ${ }^{1}$ It is thought that the survival rates of postextraction implants are high and comparable to those implants placed in healing sites. ${ }^{11}$ Considerable bone loss has been shown to be associated with socket healing. Bone healing and remodeling affect both the inside of the socket and the outer surface. The edentulous site shrinks and bone resorbes. ${ }^{1,12}$ Most studies contained only data on implant loss, but did not provide a useful information on implant failure or hard and soft tissue changes. ${ }^{7}$

The present study demonstrated that the amount of bone loss was not different between a delay placed implant and a fresh socket placed implant in the same conditions. By the time, a continuous marginal bone loss has been observed in the both groups. A study showed that crestal bone response to immediate or delayed placement of an implant into an 
another important factor for crestal bone stability. ${ }^{17}$ In animal studies, it was observed that extraction sockets were not associated with dimensional changes in implants immediately placed into the center of fresh sockets and hence, the buccal aspect of the implant during tissue modeling became exposed to the mucosa. ${ }^{18}$ When the implant shoulder was located in a buccal position in the fresh extraction socket, three times as much tissue recession occurred as when the implant had been placed in a lingual position. ${ }^{17}$ Placing the implants in a lingual position into the extraction socket, the large buccal gap that occurred between the titanium device and the bone wall during healing became filled with bone. The implant thus became fully integrated into bone. ${ }^{19}$ The gap between the implant and socket wall is another important factor for a proper the implant-bone integration. In fresh extraction socket implants, the width of the gap between the implant surface and the bone walls at the time of implant placement represents a critical point for bone healing, since as the gap widens, the amount of bone-implant contact decreases, and the point of the highest bone-implant contact shifts apically. ${ }^{20}$ When a gap between the implant and the socket exceeds $1 \mathrm{~mm}$, bone bridging may be incomplete or delayed and osseointegration may be compromised. ${ }^{1}$ Filling these gaps with bone substitute materials is mandatory. Some studies suggested a rough surface and microthreads at the implant neck not only reduce crestal bone loss but also improve early biomechanical adaptation to immediate loading. ${ }^{21-23}$

Placing implants in the fresh socket has several advantages. Surgery was simplified and directional insertion could be done. It was also thought that immediate placement of implants could prevent bone resorption and remodeling of the socket occurs. Placement of the implant at the palatal aspect of the socket seems to be important to prevent gingival recession. ${ }^{1}$ There is a suggestion that immediate and immediate-delayed implants may be at a higher risk of implant failure and complications than delayed implants, on the other hand the esthetic outcome might be better when placing implants just after tooth extraction. There is not enough reliable evidence supporting or refuting the need for augmentation procedures at immediate implants placed in fresh extraction sockets or whether any of the augmentation techniques is superior to the others. ${ }^{24}$

\section{CONCLUSION}

Comparison of marginal bone loss in fresh socket placed implants and delay implants did not show a significant difference. Also a continuous bone resorption was observed over the time in the both groups. It may be needed to investigate other success criteria in fresh socket placed implants for making an appropriate treatment plane.

\section{REFERENCES}

1. Kahnberg KE. Immediate implant placement in fresh extraction sockets: a clinical report. Int J Oral Maxillofac Implants 2009 Mar-Apr;24(2):282-288.

2. Huber S, Rentsch-Kollàr A, Grogg F, Katsoulis J, Mericske R. A 1-year controlled clinical trial of immediate implants placed in fresh extraction sockets: stability measurements and crestal bone level changes. Clin Implant Dent Relat Res 2012 Aug;14(4):491-500.

3. Lazzarra RJ. Immediate implant placement into extraction sites: surgical and restorative advantages. Int J Periodontics Restorative Dent 1989;9(5):333-343.

4. Watzek G, Haider R, Mensdorf-Pouilly N, Haas R. Immediate and delayed implantation for complete restoration of the jaw following extraction of all residual teeth: a retrospective study comparing different types of serial immediate implantation. Int J Oral Maxillofac Implants 1995 Sep-Oct;10(5):561-567.

5. Kan JYK, Rungchassaeng K, Lozada J. Immediate placement and provisionalization of maxillary anterior single implants: 1year prospective study. Int J Oral Maxillofac Implants 2003 Jan-Feb;18:31-39.

6. Lang NP, Pun L, Lau KY, Li KY, Wong MC. A systematic review on survival and success rates of implants placed immediately into fresh extraction socketsafter at least 1 year. Clin Oral Implants Res 2012 Feb;23(Suppl 5):39-66.

7. Ortega-Martínez J, Pérez-Pascual T, Mareque-Bueno S, Hernández-Alfaro F, Ferrés-Padró E. Immediate implants following tooth extraction. A systematic review. Med Oral Patol Oral Cir Bucal 2012 Mar 1;17(2):e251-e261.

8. Chen ST, Darby IB, Reynolds EC. A prospective clinical study of non-submerged immediate implants: clinical outcomes and esthetic results. Clin Oral Implants Res 2007 Oct;18:552-562.

9. Quirynen M, Van Assche N, Botticelli D, Berglundh T. How does the timing of implant placement to extraction affect outcome? Int J Oral Maxillofac Implants 2007;22(Suppl): 203-223.

10. Cardaropoli G, Araújo M, Lindhe J. Dynamics of bone tissue formation in tooth extraction sites. An experimental study in dogs. J Clin Periodontol 2003 Sep;30(9):809-818.

11. Chen ST, Beagle J, Jensen SS, Chiapasco M, Darby I. Consensus statements and recommended clinical procedures regarding surgical techniques. Int J Oral Maxillofac Implants 2009;24 Suppl:272-278.

12. Covani U, Bortolaia C, Barone A, Sbordone L. Buccolingual crestal bone changes after immediate and delayed implant placement. J Periodontol 2004 Dec;75(12):1605-1612.

13. Block MS, Mercante DE, Lirette D, Mohamed W, Ryser M, Castellon P. Prospective evaluation of immediate and delayed provisional single tooth restorations. J Oral Maxillofac Surg 2009 Nov;67(11 Suppl):89-107

14. Schropp L, Kostopoulos L, Wenzel A, Isidor F. Clinical and radiographic performance of delayed-immediate single-tooth implant placement associated with peri-implant bone defects. A 2-year prospective, controlled, randomized follow-up report. J Clin Periodontol 2005 May;32(5):480-487.

15. Botticelli D, Renzi A, Lindhe J, Berglundh T. Implants in fresh extraction sockets: a prospective 5-year follow-up clinical study. Clin Oral Implants Res 2008 Dec;19(12):1226-1232. 
16. Botticelli D, Berglundh T, Lindhe J. Hard-tissue alterations following immediate implant placement in extraction sites. J Clin Periodontol 2004 Oct;31(10):820-828.

17. Tomasi C, Sanz M, Cecchinato D, Pjetursson B, Ferrus J, Lang NP, Lindhe J. Bone dimensional variations at implants placed in fresh extraction sockets: a multilevel multivariate analysis. Clin Oral Implants Res 2010 Jan;21(1):30-36.

18. Araújo MG, Lindhe J. Dimensional ridge alterations following tooth extraction. An experimental study in the dog. J Clin Periodontol 2005 Feb;32(2):212-218.

19. Araújo MG, Wennström JL, Lindhe J. Modeling of the buccal and lingual bone walls of fresh extraction sites following implant installation. Clin Oral Implants Res 2006 Dec;17(6):606-614.

20. Akimoto K, Becker W, Persson R, Baker DA, Roher MD, O’Neal RB. Evaluation of titanium implants placed into simulated extraction sockets: a study in dogs. Int J Oral Maxillofac Implants 1999 May-Jun;14(3):351-360.

21. Shin Y-K, Han C-H, Heo S-J, Kim S, Chun H-J. Radiographic evaluation of marginal bone level around implants with different neck design after 1 year. Int J Maxillofac Implants 2006 SepOct;21(5):789-794.

22. de Sanctis M, Vignoletti F, Discepoli N, Muñoz F, Sanz M. Immediate implants at fresh extraction sockets: an experimental study in the beagle dog comparing four different implant systems. Soft tissue findings. J Clin Periodontol 2010 Aug 1;37(8):769-776.

23. Crespi R, Capparé P, Gherlone E, Romanos GE. Immediate versus delayed loading of dental implants placed in fresh extraction sockets in the maxillary esthetic zone: a clinical comparative study. Int J Oral Maxillofac Implants 2008 JulAug;23(4):753-758.

24. Esposito M, Grusovin MG, Polyzos IP, Felice P, Worthington HV. Timing of implant placement after tooth extraction: immediate, immediate-delayed or delayed implants? A Cochrane systematic review. Eur J Oral Implantol 2010 Autumn;3(3): 189-205.

\section{ABOUT THE AUTHORS}

\section{Reza Tabrizi (Corresponding Author)}

Assistant Professor, Department of Oral and Maxillofacial Surgery Shiraz University of Medical Science, Fars, Iran (Islamic Republic of), Phone: 00989125850829, e-mail: tabmed@gmail.com

\section{Mohammadreza Azizi}

Student, Department of Oral and Maxillofacial Surgery, Shiraz University of Medical Science, Fars, Iran (Islamic Republic of) 